\title{
Sensitization mechanism of cells with TDP1 inhibitors to the action of topotecan
}

\author{
Nadezhda S. Dyrkheeva \\ Institute of Chemical Biology and \\ Fundamental Medicine SB RAS \\ Novosibirsk, Russia \\ IrinaV. Il'ina \\ N.N. Vorozhtsov Novosibirsk Institute \\ of Organic Chemistry SB RAS \\ Novosibirsk, Russia \\ Nikolay S. Li-Zhulanov \\ N.N. Vorozhtsov Novosibirsk Institute \\ of Organic Chemistry SB RAS \\ Novosibirsk, Russia
}

\author{
Anastasiya A. Malakhova \\ Federal Research Centre, Institute of \\ Cytology and Genetics SB RAS \\ Novosibirsk, Russia \\ Sergey P. Medvedev \\ Federal Research Centre, Institute of \\ Cytology and Genetics SB RAS \\ Novosibirsk, Russia \\ Suren M. Zakian \\ Federal Research Centre, Institute of \\ Cytology and Genetics SB RAS \\ Novosibirsk, Russia
}

\author{
Konstantin P. Volcho \\ N.N. Vorozhtsov Novosibirsk Institute \\ of Organic Chemistry SB RAS \\ Novosibirsk, Russia \\ Nariman F. Salakhutdinov \\ N.N. Vorozhtsov Novosibirsk Institute \\ of Organic Chemistry SB RAS \\ Novosibirsk, Russia \\ Olga I. Lavrik \\ Institute of Chemical Biology and \\ Fundamental Medicine SB RAS \\ Novosibirsk, Russia
}

\begin{abstract}
A novel structural type of monoterpenederived tyrosyl-DNA phosphodiesterase 1 (TDP1) inhibitors have been discovered that was synthesized through preliminary isomerization of $(+)-3$-carene to $(+)-2$-carene followed by reaction with heteroaromatic aldehydes. All compounds inhibit the TDP1 enzyme at micro- and submicromolar levels with the most potent compound having an IC50 value of $0.65 \mu \mathrm{M}$. TDP1 is an important DNA repair enzyme and promising inhibition target for the development of new chemosensitizing agents. Panel of isogenic clones of the HEK293FT cell line knockout for TDP1 gene was created using the CRISPR-Cas9 system. Cytotoxic effect of topotecan (Tpc) and non-cytotoxic compounds of the novel structural types were investigated separately and jointly on the TDP1 gene knockout cells. For two TDP1 inhibitors synergistic effect was observed with Tpc on HEK293FT parental wildtype cells but was not found on TDP1-/- cells. Thus, it is likely that the synergistic effect with Tpc for these compounds on HEK293FT cell growth is caused by TDP1 activity inhibition. The synergy was also found for these compounds on the cancer cell lines. Such an approach in chemotherapy, with a sensitizing effect when adding a non-cytotoxic drug, can enhance the efficacy of currently used pharmaceuticals and concomitantly reduce the potential for toxic side effects.
\end{abstract}

Keywords - tyrosyl-DNA phosphodiesterase 1; TDP1 gene knockout cells; synergy; topotecan; inhibitor

\section{Introduction}

There are a number of problems arising in the course of chemotherapeutic treatment for oncological diseases, namely, low efficiency of chemotherapy, the resistance of malignant tumors to drugs, numerous side effects and high toxic load on the body. The cytotoxic effect of chemotherapy is caused by DNA damage, and the ability of cells to recognize and repair DNA lesions results in resistance. The design of new compounds that inhibit DNA repair enzymes is a promising strategy for potentiating the cytotoxicity of DNA damaging agents in clinical use. The enzymes involved in DNA repair, for example, tyrosylDNA-phosphodiesterase 1 (TDP1), are promising therapeutic targets for the treatment of cancer [1-4]. Being one of the DNA repair enzymes, TDP1 plays a key role in the removal of DNA lesions, including those resulting from the action of DNA topoisomerase I (TOP1) inhibitors. TOP1 is an essential enzyme that regulates DNA topology by reducing DNA supercoiling. TOP1 introduces a transient single-strand break in DNA, enabling the broken strand to rotate around the TOP1-bound DNA during fundamental cellular events such as replication, transcription, and repair [5]. TOP1 inhibitors, for example, topotecan (Tpc), are important chemotherapeutic agents that stabilize the cleavage complex TOP1-DNA, thereby inducing significant DNA damage leading to cell death [6]. However, TDP1 removes lesions caused by TOP1 inhibitors thus reducing their efficacy and leading to resistance [7-9]. A promising approach to increase the selectivity and potency of TOP1 inhibitors to cancer cells is to combine them with TDP1 inhibitors. In recent years, TDP1 inhibitors of various types have been discovered [1-4] including natural product derivatives. Monoterpenes, which have a unique diverse structure and are inexpensive, available and often enantiomerically pure is an attractive renewable raw material for the development of physiologically active agents. Some of the derivatives exhibit analgesic, antiviral, neuroprotective and antitumor properties [10]. Monoterpene (+)-3-carene is one of the main components of turpentine. In the current work we have studied anti-TDP1 activity of $(+)-3$-carene derivatives for the first time. For this purpose, two sets of $(+)-3$-carene derivatives of new structural types were synthesized and tested as potent TDP1 inhibitors.

To clarify the inhibitors' action on the cellular level a panel of HEK293FT TDP1 knockout isogenic clones was created using the CRISPR-Cas9 approach. The cytotoxic effect of Tpc and the new TDP1 inhibitors was measured separately and jointly.

\section{Results}

We tested 15 compounds synthesized starting from (+)3 -carene for their TDP1 inhibitory properties by measuring their $\mathrm{IC}_{50}$ (half maximal inhibitory concentration) values using a real-time fluorescent oligonucleotide biosensor [11]. This biosensor demonstrated high sensitivity and specificity for detection of TDP1. The $\mathrm{IC}_{50}$ values for substances with heterocyclic substituents varied from $0.65 \mu \mathrm{M}$ to $28 \mu \mathrm{M}$.

To examine the potential impact of TDP1 knockout on cell survival under the treatment by the synthesized compounds, we generated HEK293FT TDP1 deficient 
(TDP1-/-) cell line. Using paired gRNA CRISPR-Cas9 strategy and polymerase chain reaction (PCR) screening of cell clones we obtained clones containing deletions in the first protein coding exon of TDP1 gene. After sequencing and first treating of TDP1 proficient (wild-type) and TDP1 deficient (TDP1-/-) cells with increasing concentrations of Tpc we selected one clone for subsequent work as it has homozigous deletion causing open reading frame shift in both alleles of the TDP1 gene. It was subsequently screened by biochemical assay for 3'-phosphotyrosyl cleavage activity to identify detectable TDP1 activity in the cell extract. There was no established cleavage activity in the TDP1-/- cell extract in contrast to control WT cell extract and purified TDP1. Than we analyzed, using colorimetric test, the relative amount of viable cells after treating HEK293FT WT and TDP1 deficient (TDP1-/-) cells with increasing concentrations of Tpc for 72 hours. TDP1 knockout reduced cells viability after Tpc treatment, thus HEK293FT TDP1-/- cells where more sensitive to Tpc, thus reflecting the contribution of TDP1 on cell survival.

An analysis of the intrinsic cytotoxicity for the synthesized compounds was performed on HEK293FT WT and TDP1 deficient (TDP1-/-) cell lines by colorimetric test. We tested several compounds with the highest TDP1 inhibitory activity. Cytotoxicity is absent or insignificant in the range of studied concentrations $(0.08-100 \mu \mathrm{M})$ for all the compounds for both cell lines, which is of great importance in terms of the absence of additional side effects of cancer therapy.

TDP1 can work against the action of TOP1 poison such as Tpc due to the ability to cleave the TOP1-DNA stalled complex. Thus, TDP1 inhibition could increase the efficiency of TOP1 poisons. Next, we checked the influence of the most effective TDP1 inhibitors on the cytotoxic effect of Tpc on the HEK293FT WT and mutant cell lines. We tested HEK293FT cells viability after treatment with $11 \mathrm{~h}$ and $12 \mathrm{k}$ alone and with Tpc. There is suppressed cell growth in the joint presence of TDP1 inhibitor and Tpc on WT cells and no effect of Tpc presence in TDP1 deficient cells (Fig. 1). Thus, the synergistic action of Tpc in conjunction with $11 \mathrm{~h}$ and $12 \mathrm{k}$ on HEK293FT WT cells is likely due to the TDP1 inhibition making this enzyme the main target of action.
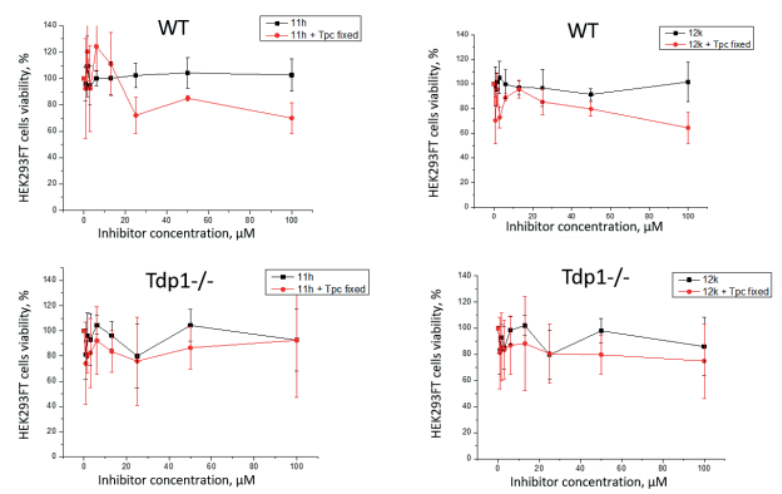

Fig. 1. TDP1 inhibitors' (11h and 12k) cytotoxicity with topotecan (Tpc) on HEK293FT WT and TDP1-/- cells.

\section{Conclusions}

We found a new class of TDP1 inhibitors with activity in the low micromolar or submicromolar concentrations, it was synthesized based on readily available monoterpene 3carene. All the tested derivatives exhibited low intrinsic cytotoxicity when tested on various cell lines (data not shown). We created a panel of isogenic clones of HEK293FT cell line knockout for TDP1 using the CRISPRCas9 system and selected one clone for the experiments. We investigated the cytotoxic effect of Tpc and two TDP1 inhibitors separately and jointly on TDP1 gene knockout cells. HEK293FT TDP1-/- cells were more sensitive to Tpc compared to WT cells. For both tested compounds we observed cell growth suppression in the presence of Tpc only for WT cells but not for the TDP1 knockout cells. Thus, it is likely that the synergistic effect with Tpc on HEK293FT cell growth is only caused by TDP1 activity inhibition by these two new compounds minimizing any off-target effects. Two most promising compounds with the best combination of TDP1 inhibition exhibited $\mathrm{IC}_{50}$ values of $0.75 \mu \mathrm{M}$ and $1.20 \mu \mathrm{M}$ and demonstrate low cytotoxicity at concentrations up to $100 \mu \mathrm{M}$ and enhancement of the Tpc efficacy on the cells. Thus, these new inhibitors are promising candidates for adjuvant therapy, mainly due to the absence of additional toxic load.

\section{ACKNOWLEDGMENT}

The work was supported by RFBR (project no. 19-415$540002)$

\section{REFERENCES}

[1] Huang, S.N.; Pommier Y., Marchand C. Tyrosyl-DNA Phosphodiesterase 1 (Tdp1) inhibitors. Expert Opin. Ther. Pat. 2011, 21, 1285-1292.

[2] Laev, S.S.; Salakhutdinov, N.F.; Lavrik O.I. Tyrosyl-DNA phosphodiesterase inhibitors: Progress and potential. Bioorg. Med. Chem. 2016, 24, 5017-5027.

[3] Zakharenko, A.; Dyrkheeva, N.; Lavrik, O. Dual DNA topoisomerase 1 and tyrosyl-DNA phosphodiesterase 1 inhibition for improved anticancer activity. Med Res Rev.; 2019, 39, 14271441.

[4] Kawale, A.S.; Povirk, L.F. Tyrosyl-DNA phosphodiesterases: rescuing the genome from the risks of relaxation. Nucleic Acids Res. 2018, 46, 520-537.

[5] Interthal, H.; Pouliot, J.J.; Champoux, J.J. The tyrosyl-DNA phosphodiesterase Tdp1 is a member of the phospholipase D superfamily. PNAS 2001, 98, 12009-12014.

[6] Pommier, Y. Topoisomerase I inhibitors: camptothecins and beyond. Nat. Rev. Cancer 2006, 6, 789-802.

[7] Dexheimer, T.S.; Antony, S; Marchand, C.; Pommier, Y. TyrosylDNA phosphodiesterase as a target for anticancer therapy. AntiCancer Agent ME 2008, 8, 381-389.

[8] Beretta, G.L.; Cossa, G.; Gatti, L.; Zunino, F.; Perego P. TyrosylDNA Phosphodiesterase 1 targeting for modulation of camptothecin-based treatment. Curr. Med. Chem. 2010, 17, 15001508 .

[9] Ledesma, F.C.; El Khamisy, Sh.F.; Zuma, M. C.; Osborn, K.; Caldecott, K.W. A human 5'-tyrosyl DNA phosphodiesterase that repairs topoisomerase-mediated DNA damage. Nature 2009, 461, 674-678.

[10] Salakhutdinov, N.; Volcho, K.; Yarovaya, O. Monoterpenes as a renewable source of biologically active compounds. Pure Appl. Chem. 2017, 89, 1105-1117.

[11] Zakharenko, A.L.; Khomenko, T.M.; Zhukova, S.V.; Koval, O.A.; Zakharova, O.D.; Anarbaev, R.O.; Lebedeva, N.A.; Korchagina, D.V.; Komarova, N.I.; Vasiliev, V.G.; Reynisson, J.; Volcho, K.P.; Salakhutdinov, N.F.; Lavrik, O.I. Synthesis and biological evaluation of novel tyrosyl-DNA phosphodiesterase 1 inhibitors with a benzopentathiepine moiety. Bioorg. Med. Chem. 2015; 23, 2044-2052. 